\title{
Atomic diffusion during red giant evolution
}

\author{
G. Michaud ${ }^{1,2}$, J. Richer ${ }^{2}$, and O. Richard ${ }^{3}$ \\ 1 LUTH, Observatoire de Paris, CNRS, Université Paris Diderot, 5 Place Jules Janssen, 92190 Meudon, France \\ 2 Département de Physique, Université de Montréal, Montréal, PQ, H3C 3J7, Canada \\ e-mail: michaudg@astro.umontreal.ca,jacques.richer@umontreal.ca \\ ${ }^{3}$ Université Montpellier II - GRAAL, CNRS - UMR 5024, place Eugène Bataillon, 34095 Montpellier, France \\ e-mail: 0livier.Richard@graal .univ-montp2 . fr
}

Received 14 April 2009 / Accepted 15 December 2009

\section{ABSTRACT}

\begin{abstract}
Context. Atomic diffusion has been found to play a role during most stellar evolution stages.
Aims. Its effect is studied during the relatively rapid red giant (RG) phase to determine the concentration variations it leads to and at what accuracy level it can be safely neglected.

Methods. A model calculated with atomic diffusion to the helium flash is compared to one calculated without any atomic diffusion and to one calculated with atomic diffusion up to a point on the subgiant branch well past the turnoff but without diffusion thereafter. Results. For stars with a metallicity of $Z=10^{-4}$, it was found that the mass of the helium core at which the He flash occurs is $0.0026 M_{\odot}$ larger in the presence of atomic diffusion. The difference decreases to $0.0017 M_{\odot}$ as metallicity is increased to $Z=0.02$. Radiative accelerations are found to play an interesting role around the hydrogen burning shell. The atomic diffusion of ${ }^{4} \mathrm{He}$ is also shown to lead to a larger $\mu$ inversion than ${ }^{3} \mathrm{He}$ burning. Its potential role in mixing between the burning shell and the surface convection zone is investigated.

Conclusions. Whether one may neglect atomic diffusion during the RG phase depends on the required accuracy. It is not so negligible as one may have expected but still only reduces by about 0.02 dex the luminosity of the RG branch bump. The way it modifies the mass of the core when the flash occurs depends on metallicity.
\end{abstract}

Key words. diffusion - stars: evolution - stars: Population II - stars: interiors - stars: abundances

\section{Astrophysical context}

In previous stellar evolution models including the effect of atomic diffusion that were calculated to the end of the the red giant (RG) phase (for instance Proffitt \& VandenBerg 1991; Cassisi et al. 1997, 1998) it was usually not made clear what effect, if any, atomic diffusion has during the RG phase itself. Those studies mainly looked at the end effect and linked any effect of atomic diffusion mainly to what happened before the RG phase. However, is it necessary to take the trouble to include atomic diffusion during the RG phase? Evolution on the giant branch is less demanding when done with a nonLagrangian method such as that developed by Eggleton (1971); is it necessary to include diffusion equations using that method? Furthermore previous studies usually only included gravitational settling of $\mathrm{He}$ and sometimes $\mathrm{CNO}$ and $\mathrm{Fe}$ assumed representative of all other metals. Most previous calculations were done including the effect of composition variations on opacity by interpolating in tables as a function of $Y$ and $Z$. To what extent is that justified when metal abundance depends on CNO abundance, $\mathrm{Fe}, \ldots$ whose composition should vary independently and modify opacity differently. The calculations described in this paper are the first to include radiative accelerations $\left(g_{\mathrm{rad}}\right)$ as well as the effect of the composition changes of individual metals on opacity as they are affected by diffusion and nuclear reactions. They also take into account the interaction between diffusing $\mathrm{H}$, $\mathrm{He}$ and metals. This is important around the $\mathrm{H}$ burning shell where nuclear reactions tend to establish extreme composition gradients. As seen below, the stronger electrostatic interaction between $\mathrm{He}$ and highly ionized metals tends to drag metals with He diffusing from the He core. One of our aims is to determine which terms are important in the diffusion equation during the RG phase to justify what can be neglected. Are $g_{\text {rad }}$, the dragging by metals, gravitational settling of He or of metals dominant?

The effect of the gravitational settling of $\mathrm{He}$ and metals on the luminosity function bump has been carefully evaluated by Cassisi et al. (1997, 1998). This is important in relation to age determinations of globular clusters. One may ask what effect a more complete treatment of atomic diffusion may have on it.

On the other hand, the origin of abundance variations on the red giant branch (RGB) is not yet explained satisfactorily. Denissenkov \& VandenBerg (2003) used observed Li, C and $\mathrm{N}$ surface abundances and $\mathrm{C}$ isotopic ratios to constrain extra mixing processes in RGB stars. Sweigart \& Mengel (1979) discussed the importance of a $\mu$ gradient inversion around the $\mathrm{H}$ burning shell in order to understand the abundance variations seen at the surface of RGB stars. They mainly studied the effect of partial CNO burning above the $\mathrm{H}$ burning shell and how this was a function of $Z$. Such inversions modify the stability of the fluid and so the potential penetration of mixing processes with nuclear processed material. Indeed as the surface convection zone recedes after the first dredge-up, the $\mathrm{H}$ burning shell moves slowly toward the surface. When it reaches the region which had been homogenized by the convection zone, Eggleton et al. (2006, 2008) found in their 3-D simulation that a very small $\mu$ gradient inversion caused by ${ }^{3} \mathrm{He}$ burning just above the hydrogen burning shell is sufficient to lead to instability and mixing. It is caused by ${ }^{3} \mathrm{He}$ burning leading to an increase in the number of 
particles (Ulrich 1972) and so an inversion of the gradient of $\mu$. The mixing was later discussed by Charbonnel \& Zahn (2007a) in terms of thermohaline mixing. Charbonnel \& Zahn (2007b) argued that a magnetic field might suppress the turbulent transport caused by the $\mu$ instability and be the cause of the observed variation of ${ }^{3} \mathrm{He}$ concentration in some planetary nebulae. The importance of this process has been questioned by Denissenkov \& Pinsonneault (2008). Following Zahn (1992), they postulate the presence of strong horizontal turbulence linked to rotation and argue that it would wipe out the weak vertical turbulence caused by the small $\mu$ inversion. They also argue that the systematics of abundance anomalies on the RGB goes against the anomalies to be expected from this process.

Our results are not able to settle this issue. In fact, they may further complicate the situation: does atomic diffusion create $\mu$ inversions larger than created by ${ }^{3} \mathrm{He}$ and what systematic variation would this lead to on the RGB?

After a brief description of the calculations (Sect. 2), global structural properties are described (Sect. 3) and then the effect of atomic diffusion on the luminosity function bump is evaluated (Sect. 4). We next compare (Sect. 5) the internal properties of the three models around the region where the flash ignites and take a closer look at the transport processes around the $\mathrm{H}$ burning shell (Sects. 6 and 7). The extent to which atomic diffusion around and above the burning shell is responsible for the development of an inverted $\mu$ gradient leading to the possibility of mixing between the burning shell and the convection zone depends on the interaction between developing anomalies and turbulent transport. How large does the turbulent diffusion coefficient need to be in order to eliminate the small $\mu$ gradients that ${ }^{3} \mathrm{He}$ burning and atomic diffusion lead to (Sect. 7.1)?

\section{Calculations}

Stellar evolution calculations were carried out to the horizontalbranch (HB), starting from the pre-main-sequence, with diffusion turned on at the zero age main-sequence, as described in Michaud et al. (2007). They were calculated from first principles with the mixing length calibrated using the Sun (Turcotte et al. 1998); all aspects of atomic diffusion transport are treated in detail. These models are called models with diffusion as in Michaud et al. (2007). Using the same code, models were calculated without atomic diffusion and are called models without diffusion. Another model was calculated with atomic diffusion from the zero age main-sequence to a point on the subgiant branch well passed the turnoff but without atomic diffusion after that phase. It is called intermediate model. A few additional models were also calculated to evaluate the impact of variations in input physics. A model with diffusion was calculated forcing the surface convection zone to incorporate the tiny convection zones that occur during dredge-up; this is approximately equivalent to very efficient semi-convection. Another model was calculated with atomic diffusion but without $g_{\mathrm{rad}}$. These two models are occasionally mentioned in the text but the main models used are the models with diffusion, that without diffusion and the intermediate model. Note that, in all models, the effect of composition change on opacity is fully taken into account throughout. For instance in the model without diffusion, the opacity increase caused by the increasing $\mathrm{C}$ concentration is properly calculated as the He flash leads to increasing $\mathrm{C}$ concentration.

In preceding papers (Michaud et al. 2007, 2008) the evolution of a Pop II $M_{*}=0.8 M_{\odot}$ star with $Z=10^{-4}$ was followed taking atomic diffusion into account from the zero age mainsequence to the middle of the HB. The red giant branch (RGB) was treated in detail. Similar calculations have now also been performed for metallicities ${ }^{1}$ of $Z=10^{-3}, Z=0.004$ and $Z=$ 0.02 . They are illustrated below using mainly a $M_{*}=0.95 M_{\odot}$ star with $Z=0.004$.

\section{Structural properties}

In Fig. 1 are shown the Hertzsprung-Russell (HR) diagram and the $T_{\text {eff }}$ evolution for four models with $M=0.95 M_{\odot}$ and $Z=0.004$. The point where the intermediate model parts from the model with diffusion is indicated by $\times$. Slightly past this point, there appear small convection zones just below the surface convection zone. They are caused by a composition discontinuity as the surface convection zone becomes more massive and reaches where the concentration of metals increases because of the past effect of their gravitational settling. In the model with diffusion, the surface convection zone is assumed completely separate from those small convection zones just below it. To evaluate the effect of that assumption, the opposite assumption of complete mixing from the surface down to the bottom of the deepest of the small convection zones was used in the fourth model shown by the green line in Fig. 1. The only noticible difference is in the circle in the inset of the left panel. The green line extends approximately midway between the model with diffusion and the intermediate model. This is a very small effect and suggests that the uncertainty introduced here by semi-convection is negligible. At that point, it however becomes numerically very demanding to follow the concentration of each metal precisely; consequently, in order to simplify the calculations this is an obvious stage where one might choose to stop including diffusion ${ }^{2}$. In the HR diagram, the differences between the model with diffusion and the intermediate model are hardly distinguishable. If one looks closely, one may see, in the inset, a small difference in luminosity at the bump. Apart from that point, it is difficult to separate the two models in the HR diagram. The model without diffusion throughout is easier to distinguish in particular around the turnoff and at the bottom of the RGB. The differences between the model with diffusion and the intermediate model are very small in the evolution of the $T_{\text {eff }}$ seen in the right panel. They are however visible during the first dredge-up; we have verified that this small difference is not visible in a plot of the evolution of $L$. To facilitate reading, in this paper, ages are often given with respect to $t_{\text {hook }}$ which is defined in Fig. 1 .

In Fig. 2 is shown the evolution of the He core mass during the RG as a function of the He luminosity. The He core mass has reached its final value at the end of the curves shown. The evolution is shown for models of $0.80 M_{\odot}, Z=10^{-4}$ (two upper curves); $0.85 M_{\odot}, Z=0.001 ; M_{*}=0.95 M_{\odot}, Z=0.004$ (three curves); and $1.0 M_{\odot}, Z=0.02$. The results for the variation of the He core mass with chemical composition of models without diffusion agree within $20 \%$ with the variation obtained using Eq. (9) of Sweigart \& Gross (1978). The results for the difference in mass between models with and without diffusion for the lowest metallicity models were discussed in Michaud et al. (2007) and are also compatible with Eq. (9) of

\footnotetext{
1 In this series of papers, the relative values of the $\alpha$ elements are increased following VandenBerg et al. (2000). Models are labeled according to their original $Z$ value calculated before the $\alpha$ correction. See also Table 1 of Richard et al. (2002). This correction was not applied in the $Z=0.02$ models.

2 For instance, inspired by the method developed by Eggleton (1971), VandenBerg (1992) prefers to switch, as described in his Sect. 3, to a non-Lagrangian mesh for his RGB calculations.
} 

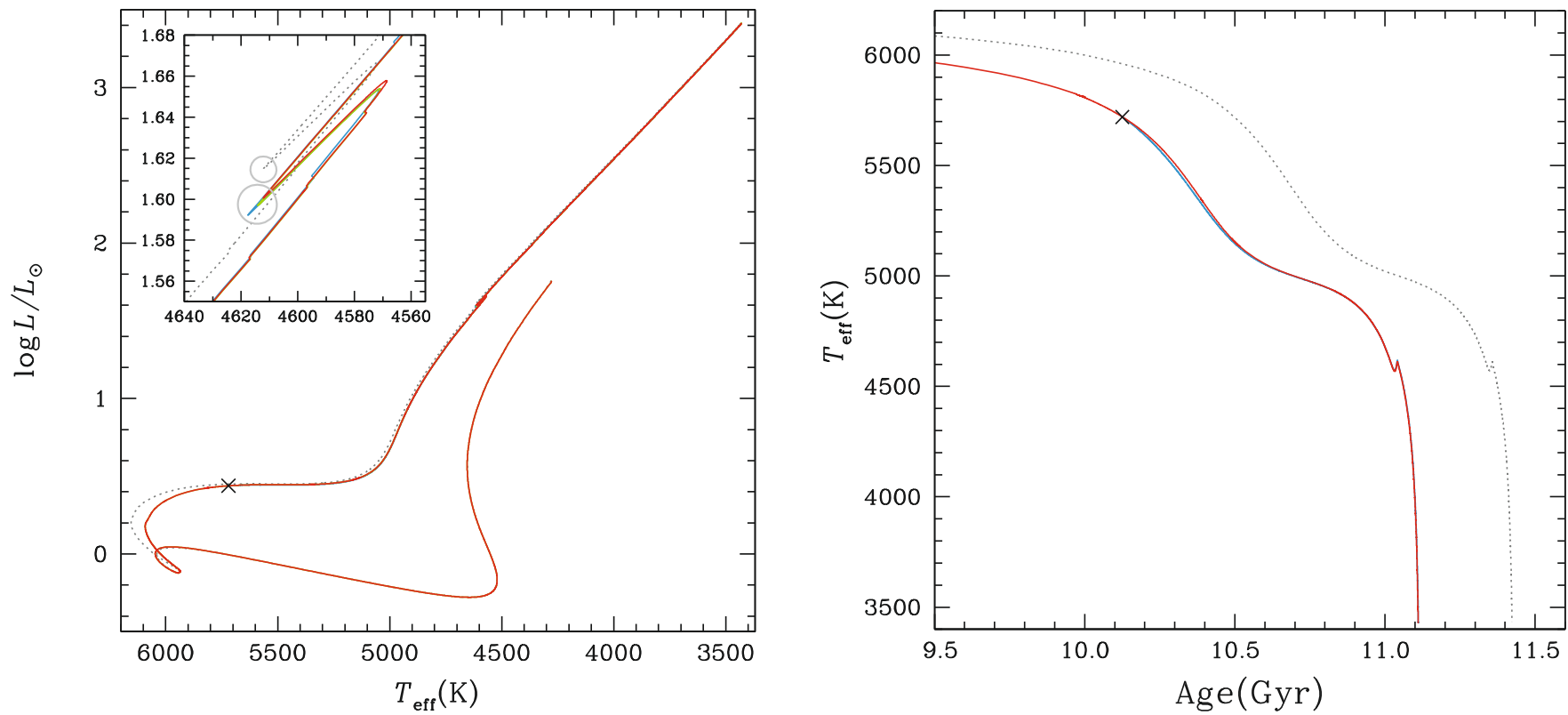

Fig. 1. Left panel: HR diagram for four models with $Z=0.004$; red: with diffusion, green: with diffusion and semi-convection during dredge-up, gray: model without diffusion, blue: intermediate model. The intermediate model parted from the models with diffusion past turnoff at the point indicated by $\times$. The largest difference is seen between the model with diffusion and the model without diffusion around the turnoff and the bottom of the giant branch. All four models are seen in the zoomed inset of the hook or bump region where one may see some effect of semi-convection. In the inset, the small breaks are a measure of the limited accuracy. The age of the models at their lowest luminosity within the circle in the inset is defined here as $t_{\text {hook}}$. It is respectively equal to 11.0415 and $11.3570 \mathrm{Gyr}$ for the models with (red) and without (gray) diffusion. To facilitate reading ages are often given with respect to $t_{\text {hook }}$ in this paper. Right panel: evolution of $T_{\text {eff }}$ for three models; the green model can not be distinguished from the red one and is not shown.

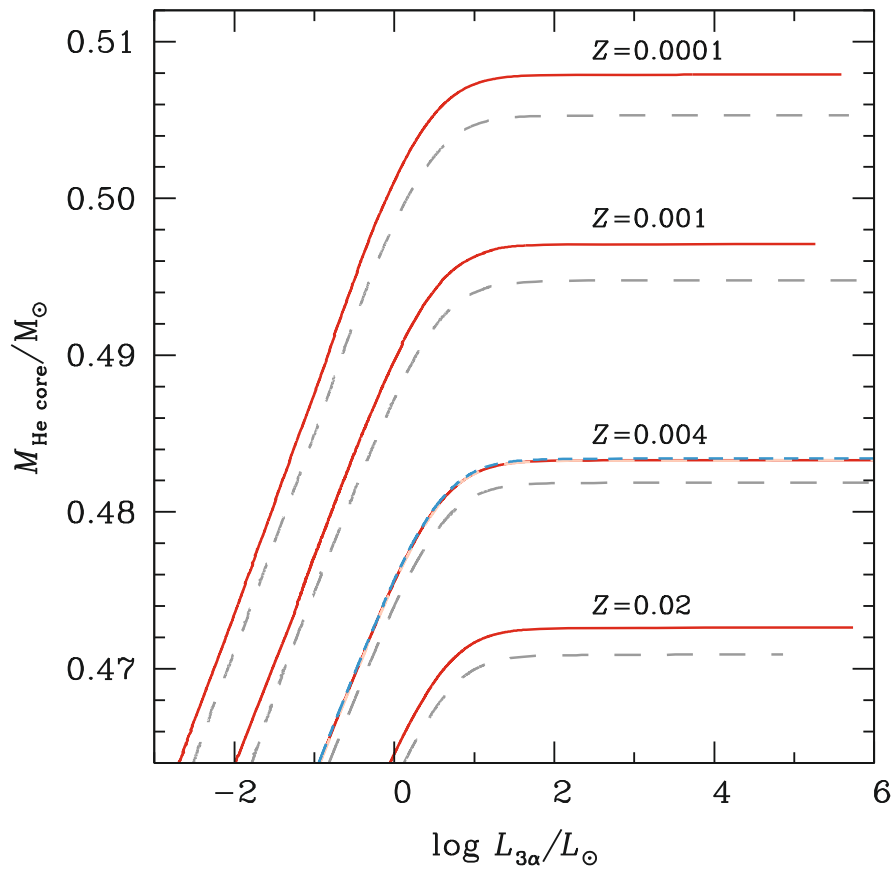

Fig. 2. Size of He core as a function of $3 \alpha$ luminosity (including a small contribution from other $\alpha$ capture reactions). The gray dashed lines are for models without diffusion while the solid red lines are for models with diffusion. Four metallicities are shown. For the $Z=0.004$ models the small-dashed blue line is for the intermediate model. Its He core mass is only marginally larger than that of the model with diffusion.

Sweigart \& Gross (1978) if one identifies the change in surface He abundance of our models with a change of $Y$ of non diffusing models. The rationale is that the remaining envelope serves as a blanket to the He core and so its opacity influences the growth of the core and so its mass. As one considers higher metallicity models the difference between the core mass of the model with diffusion and that without decreases slightly. The mass difference of the He core decreases as metallicity increases, from $0.0026 M_{\odot}$ at $Z=0.0001$ to $0.0017 M_{\odot}$ at $Z=0.02$. The intermediate model was calculated only for the $Z=0.004$ case and it has a marginally higher core mass $\left(+0.0001 M_{\odot}\right)$ than the model with diffusion ${ }^{3}$. Applying Eq. (9) of Sweigart \& Gross (1978) would lead to a much smaller metallicity dependent reduction of the core mass than $0.0017 M_{\odot}$. Stratification of the concentrations of He and $\mathrm{CNO}$ probably play a larger role.

Since the relative size of the core mass between models with diffusion and models without diffusion is a function of metallicity, it can be affected by the diffusion of metals which will be investigated in Sects. 5 and 6.

\section{Luminosity function bump}

The effect of the atomic diffusion of He and metals on the position of the luminosity function bump on the RGB was studied by Cassisi et al. (1997, 1998). They estimate how this influences age determinations using the zero age HB (ZAHB) luminosity and the RG luminosity function bump. They obtained differences of 0.07 (for models with $[\mathrm{M} / \mathrm{H}]=-2.04$ ) and 0.08 (for models with $[\mathrm{M} / \mathrm{H}]=-0.57$ ) in magnitude for the position of the bump between the models with and without diffusion. Both metallicities led to a change of the same sign. They relate that change to an opacity increase in the envelope caused by the decrease of the

\footnotetext{
3 The model with semi-convection has a core mass $0.00005 M_{\odot}$ larger and the model without $g_{\mathrm{rad}}$, a core mass $0.00005 M_{\odot}$ smaller than the model with diffusion.
} 
He abundance there ${ }^{4}$. They concluded that the effect of diffusion on the position of the bump was smaller than observational uncertainties. Since the luminosity function bump is caused by the passage of the hydrogen burning shell through the composition discontinuity left after the first dredge-up, the detailed treatment of metal diffusion and its effect on opacity has some impact on the bump luminosity but by how much?

From Fig. 1, it is seen that the luminosity difference is small for the $Z=0.004$ models. Referring to the inset, a comparison of the minima of the hook luminosity shows that the model with diffusion has a luminosity 0.014 dex smaller than the model without diffusion. The largest difference is between the model without diffusion and the intermediate model, the latter having a luminosity 0.022 dex smaller than the model without diffusion. The difference between the model without diffusion and that with semi-convection is however in between the two, 0.018 dex. It has also been verified that the model with diffusion but without $g_{\text {rad }}$ has, at the quoted accuracy, the same luminosity at the hook as that with diffusion and $g_{\mathrm{rad}}$.

For $Z=0.001$, the hook of the model without diffusion has a luminosity larger by 0.020 dex than that of the model with diffusion ${ }^{5}$. For a given mass, ZAHB models without diffusion have a 0.01 to 0.03 dex larger luminosity but the difference is larger in the cooler model we had, around $10^{4} \mathrm{~K}$.

On the $\mathrm{HB}$, the $Z=0.0001$ models with diffusion of Michaud et al. (2007) have 0.01 to 0.02 lower $\log L$ than the corresponding model without diffusion (see their Fig. 4). The hook of the model with diffusion has a luminosity smaller by 0.027 dex than that of the model without diffusion. The two effects partly cancel if one uses the difference between the two.

At the four metallicities calculated, the effect of atomic diffusion on the luminosity of the bump is small (0.01 to 0.03 dex) and partly compensated by its effect on HB luminosities $(0.02$ dex $)$ when the ratio of the two luminosities is used to factor out the uncertainty of the distance scale. Those differences are of similar size as those obtained by Cassisi et al. (1997, 1998).

\section{Properties around the flash}

In Fig. 3 are shown internal properties of the three models at the phase when He burning is developing explosively off center and the He burning shell is becoming convective. The difference between the adiabatic and radiative gradients is shown in the top panel ${ }^{6}$. A convection zone develops during the flash. It is treated in our simulations by a turbulent diffusion coefficient $\sim 10^{6} \mathrm{~cm}^{2} \mathrm{~s}^{-1}$ which is approximately $10^{6}$ times larger than the atomic diffusion coefficient of hydrogen there. As may be seen from panels (b) and (c), Fe and C are only partially mixed by the turbulent convection we impose.

Two time steps are shown for the model with diffusion in order to bracket the evolutionary stage of the models without diffusion. The first one (short dashed line) is just before the burning shell becomes convective. On panel (e), while neutrinos, as shown by the dotted lines, play a minor role, it is seen

\footnotetext{
${ }^{4}$ See the end of Sect. 2 of Cassisi et al. (1997).

5 This is based on as yet unpublished calculations.

6 The function "linlog" used in some of the figures is defined by $y=\operatorname{lin} \log (x) \Leftrightarrow x=10^{y}-10^{-y}$;
}

it is a kind of base 10 arcsinh function, useful for displaying both large and small, positive and negative values of the same function. For $|x| \gtrsim$ $8, \operatorname{lin} \log (x) \approx \operatorname{sign}(x) \cdot \log _{10}|x|$, and for $|x|<1, \operatorname{lin} \log (x) \sim x / 5$.

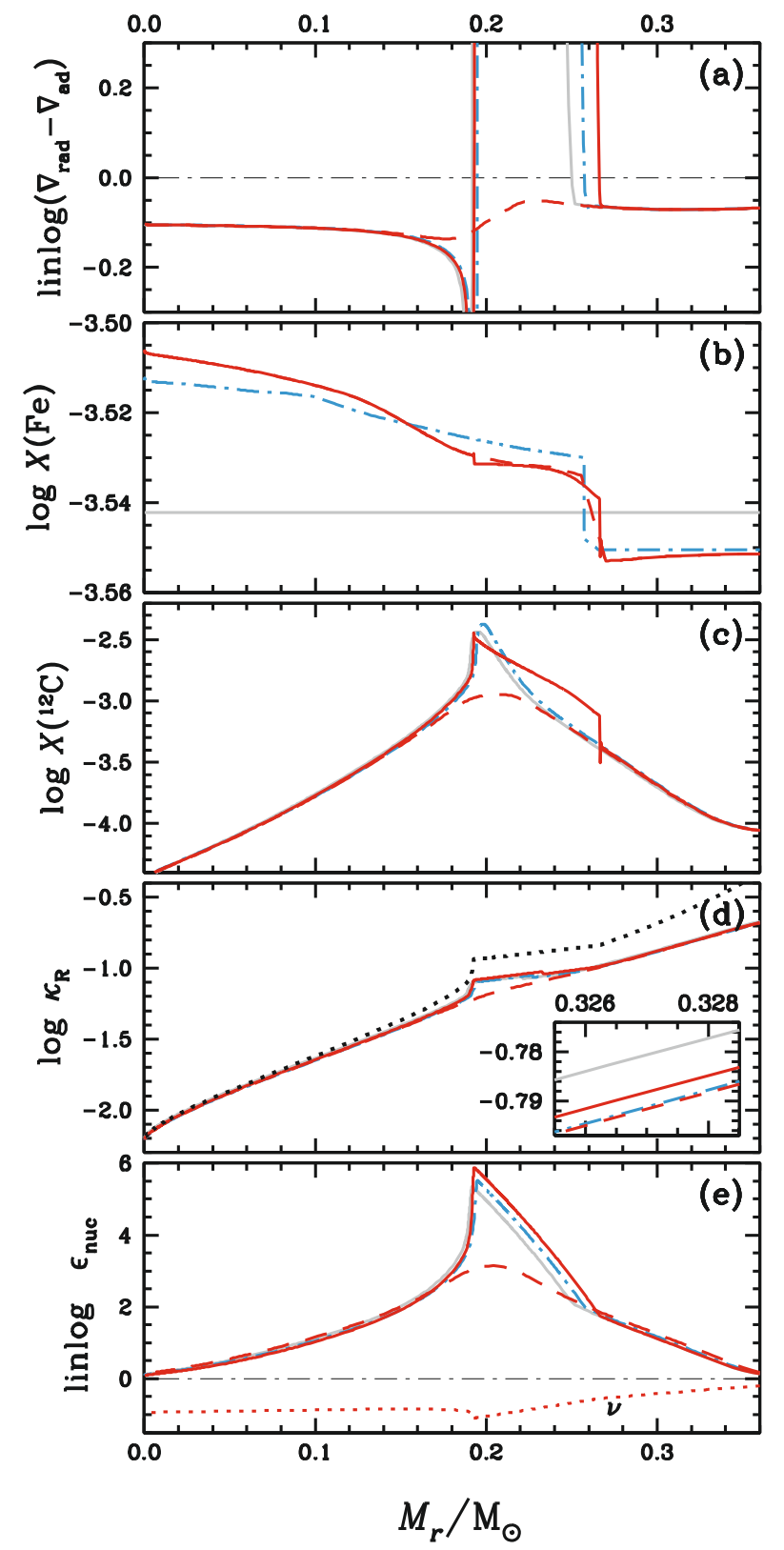

Fig. 3. Internal properties of three RG models at approximately the same He luminosity during the He flash. On panel a) is seen the convection zone generated by the flash for the model with diffusion (red lines), the model without diffusion (solid gray line) and the intermediate model (dot-dashed blue line). As the flash begins, the interior limit of the convection is at very nearly the same mass. On panels b) and c) are shown respectively the $\mathrm{Fe}$ and ${ }^{12} \mathrm{C}$ abundances. On the bottom panel, e), is shown the He nuclear energy generation for the model with diffusion for two different time steps (red lines) which bracket the energy generation of the model without diffusion and of the intermediate model. The dotted line is for the neutrino energy. On panel d), one sees that outside the flash area, the opacity (here corrected for conduction; the dotted black line represents conduction only) is nearly the same in all models. In the zoomed inset, one may see that the opacities of the model with diffusion (solid and dashed lines) and of the intermediate model (dotdashed blue line) are some $4 \%$ smaller than that of the model without diffusion (solid gray line). Especially for $M_{\mathrm{r}}<0.18 M_{\odot}$, the opacity is much smaller than the electron scattering opacity, since conduction dominates. (See footnote 5 for the definition of linlog).

that the He burning luminosity of the model without diffusion (solid gray line) and that of the intermediate model (dot-dashed blue line) are between those of the first (short dashed line) and 


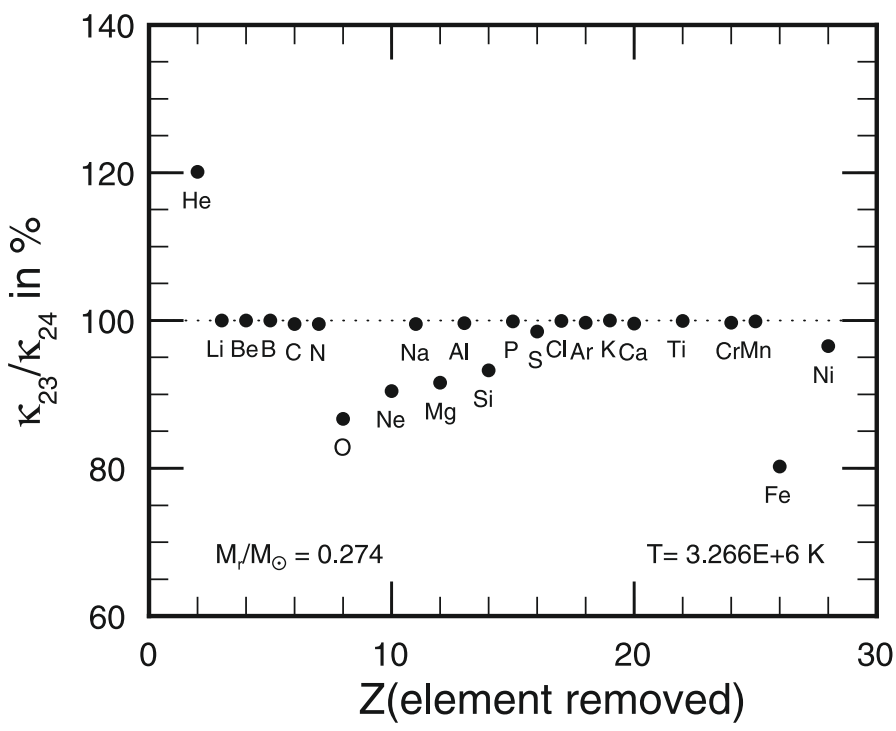

Fig. 4. Ratio of the Rosseland opacity (here including the effect of conduction) evaluated without including the spectrum of the element of atomic number $Z$ and of that obtained with the complete mix (24 elements). The concentration of each remaining species is increased to maintain normalization. It is shown at $M_{\mathrm{r}} / M_{\odot} \simeq 0.27$ which, at the evolutionary phase $\kappa$ was evaluated for this figure, is between the $\mathrm{H}$ burning shell and the bottom of the convection zone. At that $T$ and $\rho$ a mixture without $\mathrm{He}$ has a $20 \%$ larger opacity than a mixture with $\mathrm{He}$. This figure is at the phase identified as -2.9 Myr in Figs. 5 and 6.

second (solid line) models with diffusion. Comparing the two time steps with diffusion shows that they differ only by the intensity of He burning and its immediate effects on the region around $M_{\mathrm{r}} / M_{\odot}=0.2$ : little difference is seen in the opacities (panel d) between the two time steps for $M_{\mathrm{r}}<0.17 M_{\odot}$ nor for $M_{\mathrm{r}}>0.25 M_{\odot}$. If one compares the opacities of the models with and without diffusion in the region $M_{\mathrm{r}}>0.25 M_{\odot}$, one then expects the differences between them to be caused by the different physics included and not to differences in evolutionary status.

In the central region, opacity (panel d) is dominated by conduction. So, while metals (in particular Fe peak elements which are not fully ionized according OPAL's equation of state ${ }^{7}$ ) probably dominate the absorption or scattering of photons, this hardly modifies energy transport in the central region. In the region $M_{\mathrm{r}} \sim 0.27 M_{\odot}$, the effective opacity of the model without diffusion is about $2 \%$ larger than that of the intermediate model as seen in the inset in panel (d) of Fig. 3. It may also be seen in Fig. 4, that metals dominate the opacity between the $\mathrm{H}$ burning shell and the surface convection zone. However we have verified that they do not dominate deeper in, presumably because of the large role of conduction. It may seem surprising that $\mathrm{C}$ should not contribute more to opacity where the flash is occuring since it seems to produce a signature in panel (d). However the apparent signature of $\mathrm{C}$ is in fact a signature of the change in conduction (dotted black line in panel d) brought about by $T$ and $\rho$ variations caused by the flash.

Perhaps the most surprising differences are seen in panel (b). The Fe concentration of the model without diffusion (gray line) is constant as expected. The Fe concentrations in both the model with diffusion and the intermediate model vary. In the model with diffusion it is about $11 \%$ larger in the center than for $M_{\mathrm{r}}>0.3 M_{\odot}$. It is surprising that it should differ from the

\footnotetext{
7 In addition to the spectra they used to construct opacities, OPAL included the mean charge of each atomic species on its grid. See Richer et al. (1998); Rogers \& Iglesias (1992a,b); Rogers et al. (1996).
}

Fe concentration in the intermediate model. Indeed both had exactly the same Fe concentration passed turnoff. It is only beyond that evolutionary stage that they differ.

As the star climbs up the RGB, the surface convection zone becomes deeper until it reaches its deepest extension at $M_{\mathrm{r}}=0.26 M_{\odot}$. On the other hand, the $\mathrm{H}$ burning shell is at $M_{\mathrm{r}}=0.034 M_{\odot}$ when the intermediate model parts from the model with diffusion. It moves progressively outwards to $M_{\mathrm{r}}=0.48 M_{\odot}$ (see Fig. 2) when the flash occurs. As it moves outwards, it leaves behind different metal concentrations. This is looked at in more detail in Sect. 6 at about the moment when the $\mathrm{H}$ burning shell crosses the composition discontinuity which was left behind by the surface convection zone at its deepest penetration. The role of He settling between the burning shell and the bottom of the convection zone is investigated in Sect. 7 .

\section{Diffusion of metals around the $\mathbf{H}$ burning shell}

The diffusion of metals is investigated as the $\mathrm{H}$ burning shell crosses the concentration discontinuity left by the first dredge-up because of the special interest of the luminosity bump that also occurs then. However diffusion has similar effects throughout the ascent on the RGB.

In Fig. 5 are shown on the upper panels the concentrations for $\mathrm{P}, \mathrm{Cr}$ and $\mathrm{Fe}$ as a function of interior mass when the $\mathrm{H}$ burning shell (lower right panel) crosses the concentration discontinuity (the discontinuity is visible in the upper panels) left by the surface convection zone. Iron is illustrated because of its importance for opacity (see Fig. 4), Cr shows that other iron peak elements have a similar behavior as $\mathrm{Fe}$, and $\mathrm{P}$ has a behavior typical of the species from Al to Ar. There are differences in detail among species. For instance, phosphorus has a particularly strong $g_{\text {rad }}$ but those from $\mathrm{Mg}$ to Ar are all about equal to or slightly larger than gravity above the $\mathrm{H}$ burning shell.

One first notes that the difference between $X(\mathrm{P}), X(\mathrm{Cr})$ and $X(\mathrm{Fe})$ in the core of the model without diffusion and those with diffusion is by about $10 \%$. At the base of the RGB both the model with diffusion and the intermediate model had the same concentration profiles. It is the one shown here for the intermediate model since it did not vary during giant branch evolution. However in the model with diffusion, the concentration profiles vary significantly during RGB evolution and they vary differently for different species which may seem surprising. All metals increase their central concentration, due to gravitational settling and thermal diffusion. However the concentration of metals is smaller in the interval $0.15<M_{\mathrm{r}}<0.25 M_{\odot}$ in the model with diffusion than in the intermediate model and the difference is not the same for all species as may be seen here by comparing $\mathrm{P}$, $\mathrm{Cr}$ and Fe. All metals included in the calculations show slighty different patterns. These must be caused by diffusion during the RGB evolution.

Drift velocities are shown in the middle panels of Fig. 5. The drift velocities vary from one atomic species to another and are of course present only in the model with diffusion. The drift velocity of, say, Fe includes all contributions to the diffusion velocity of Fe except the purely diffusive term of Fe. It includes, in particular, the contribution coming from the interaction between $\mathrm{Fe}$ and the diffusive term of He. It so includes contributions from the interaction with the very steep $\mathrm{He}$ and $\mathrm{H}$ abundance gradients but also from gravity, thermal diffusion and radiative accelerations (shown on the lower panels of Fig. 6), the latter varying considerably from species to species. Drift velocities are largest close to the $\mathrm{H}$ burning shell. The patterns seen, in the upper panels of Fig. 5 around $M_{\mathrm{r}}=0.20 M_{\odot}$, have been generated while 
A\&A 510, A104 (2010)
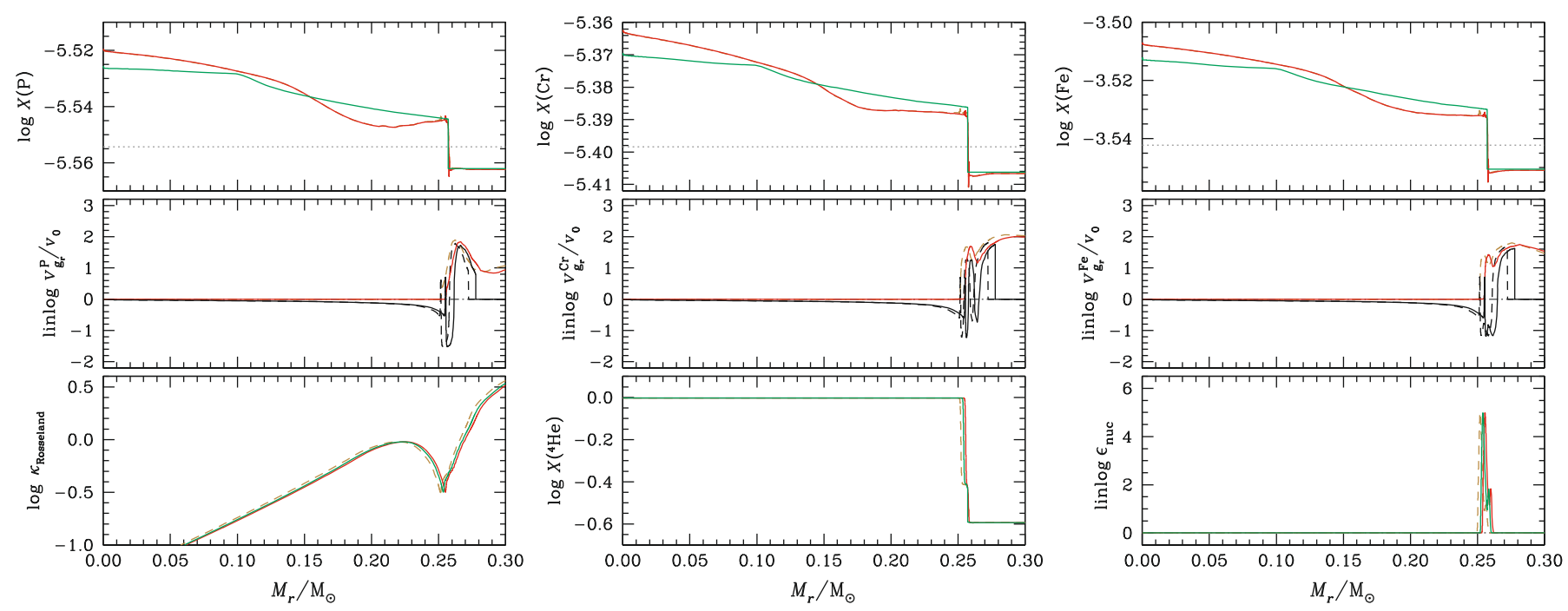

Fig. 5. Mass fractions (upper panels) and drift velocities (central panels) of $\mathrm{P}$ (left panels), $\mathrm{Cr}$ (central panels) and $\mathrm{Fe}$ (right panels) as a function of the mass interior to $r$, shortly after the maximum inward extension of the surface convection zone: more precisely when the $\mathrm{H}$ burning shell crosses the concentration jump left by the convection zone. Two ages are shown for the model with diffusion at $-7.7 \mathrm{Myr}$ (dashed brown line) and $-2.9 \mathrm{Myr}$ (solid red line) with respect to $t_{\text {hook }}$ as defined in Fig. 1. The intermediate model is shown at $-4.4 \mathrm{Myr}$ (solid green line) when its $\mathrm{H}$ burning shell is between the two shown for the model with diffusion (bottom right panel). Comparing the concentration profiles in the model with diffusion to those in the intermediate model shows the effect of atomic diffusion during the giant branch. The original values on the zero age main-sequence are the same as given by the horizontal dotted line representing the model without diffusion. In the middle row are shown, for the model with diffusion, the total drift velocities (dashed and solid black lines) and the radiative acceleration contribution to the drift velocity (dashed brown lines and solid red lines) with $v_{0}=10^{-9} \mathrm{~cm} / \mathrm{s}$. In the lower panels (same color codes as upper panels) are shown the Rosseland opacity (left), the He mass fraction (central panel) and the energy generation rate per gram (right panel). The position of the bottom of the surface convection zone is indicated by the vertical portion of the drift velocity. It is at $M_{\mathrm{r}}=0.2777 M_{\odot}$ in the diffusion model of $-2.9 \mathrm{Myr}$.
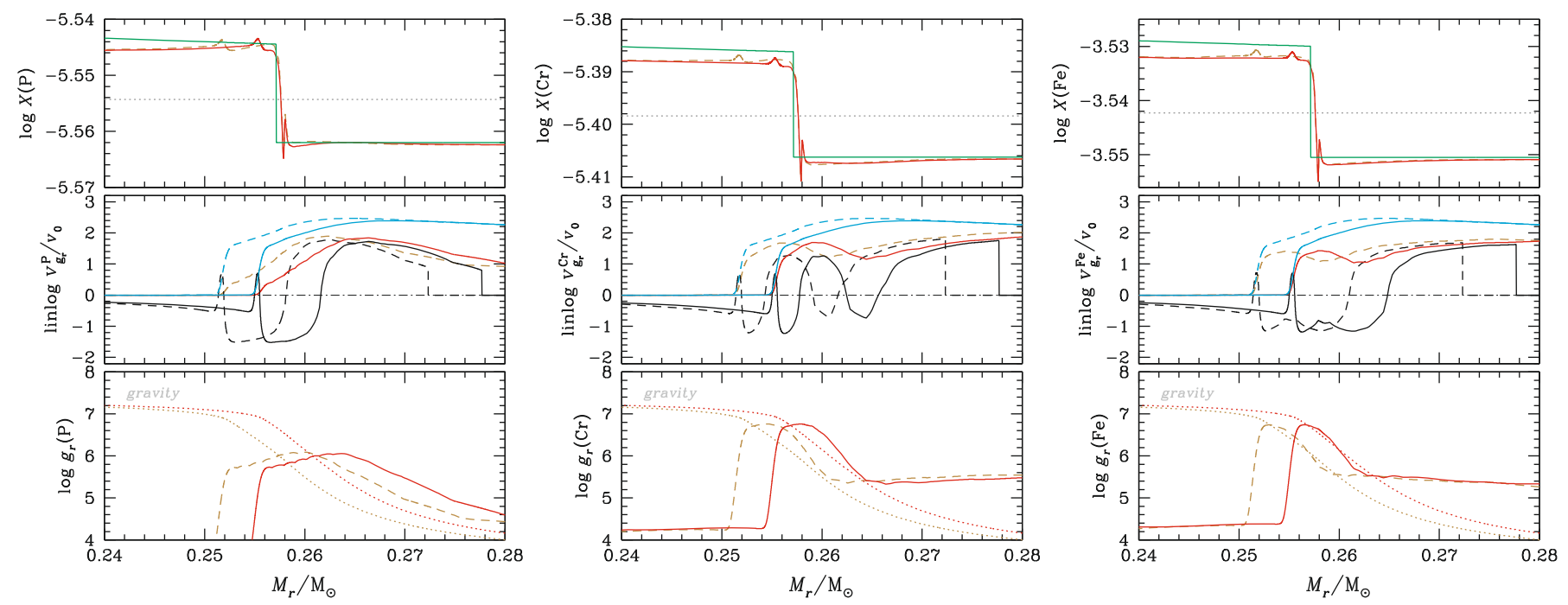

Fig. 6. The upper panels are a zoom around the $\mathrm{H}$ burning shell of mass fractions of $\mathrm{P}, \mathrm{Cr}$ and $\mathrm{Fe}$ shown in Fig. 5 at the same ages as on that figure where the curves are identified. The middle panels give (with $v_{0}=10^{-9} \mathrm{~cm} / \mathrm{s}$ ) the total drift velocity (black lines), the radiative acceleration contribution to the drift velocity (brown dashed line and red solid line) and the front velocity (see Eq. (1); blue lines) which is approximately that of matter crossing a given radius to replace $\mathrm{H}$ burned below. All three velocities are of the same order. Radiative accelerations of $\mathrm{Fe}, \mathrm{Cr}$ and $\mathrm{P}$ are shown on the lower panels where dotted lines represent gravity. The radiative accelerations are larger than gravity above the $\mathrm{H}$ burning shell for the three elements. The $g_{\mathrm{rad}}$ vary from species to species and so contribute to the different behavior of the drift velocities shown in the middle panels.

the $\mathrm{H}$ burning shell crossed that mass. Though smaller very close to the center, drift velocities still play a role there because the distances are much smaller than close to the burning shell. Drift velocities are analyzed in more detail in Fig. 6 where they are compared to a rough evaluation of the velocity of the $\mathrm{H}$ burning front, $v_{\text {front }}$, given by:

$v_{\text {front }}=\frac{L}{4 \pi r^{2} \rho c^{2} f}$ where $f$ is the fraction of mass converted into energy by $\mathrm{H}$ burning and other symbols have their usual meaning. Above the front, this velocity is approximately that of matter crossing a given radius to replace $\mathrm{H}$ burned below. This velocity is then more properly an inward velocity of matter. The upward drift velocity reduces the flux of say $\mathrm{Cr}$ carried inward by matter coming from the surface convection zone, going though the $\mathrm{H}$ burning shell and then joining the core. 
The Rosseland opacity, as modified by conduction, is shown in the lower left panel of Fig. 5. Slightly below the H burning shell, the opacity becomes dominated by conduction and so rapidly decreases. Close to the $\mathrm{H}$ burning shell metals contribute significantly to opacity as shown in Fig. 4.

A zoom of the particle transport around the $\mathrm{H}$ burning shell is shown in Fig. 6. The concentrations are shown in the upper panels at the same two time steps as in Fig. 5. In the model with diffusion, the strong $\mathrm{He}$ gradient created by the $\mathrm{H}$ burning shell causes He to move outwards dragging metals along through an extra contribution to the drift velocity of metals ${ }^{8}$. This leads to the spikes of the drift velocities seen in the middle panels (black lines at $\left.M_{\mathrm{r}} / M_{\odot} \sim 0.254\right)$. This leads in turn to the small drifting bulges seen in the upper panels. At the same time, the spikes in the drift velocity reduce the flux of metals, such as Fe, carried inwards through the burning shell (at velocity $v_{\text {front }}$ ) leading to a small underabundance of metals below the shell. The metals furthermore diffuse towards the center by gravitational settling (aided by thermal diffusion, ...). Consequently the Fe concentration is smaller in the model with diffusion than in the intermediate model for the $0.15<M_{\mathrm{r}} / M_{\odot}<0.4$ interval even if both models had the same diffusive transport for most of the evolution, from the ZAMS to well past turnoff. In the model with diffusion, gravitational settling led to an increase in $X(\mathrm{Fe})$ for $M_{\mathrm{r}}<0.15 M_{\odot}$. The concentration variations were calculated for all included species. They are shown only for $\mathrm{P}, \mathrm{Cr}$ and $\mathrm{Fe}$ but all species not involved in $\mathrm{CNO}$ nor He burning have variations of the same order, though varying in details.

One notes that the diffusion velocity of $\mathrm{P}$ caused by $g_{\text {rad }}$ (the red lines in the middle row of Fig. 6) is very different from that of $\mathrm{Cr}$ close to the $\mathrm{H}$ burning shell. For $\mathrm{Cr}$ and $\mathrm{Fe}$, in front of the frontier of the He core, this velocity very nearly equals $v_{\text {front }}$. However the total drift velocity (the black lines in the middle row of Fig. 6) is opposite but slightly smaller than the velocity of matter feeding the $\mathrm{H}$ burning shell, or $v_{\text {front }}$. This leads to a slight reduction of the $\mathrm{Cr}$ and $\mathrm{Fe}$ flux to the core. It contributes to the differences seen in the upper panels between the concentration profiles of the various species. Similar effects are seen for the other atomic species, not shown in these figures.

Later in the evolution, as the star climbs the RGB, its luminosity increases and so $v_{\text {front }}$ increases since it is proportional to $L$ (see Eq. (1)). Radiative accelerations also increase with $L$ but not gravitational settling. On figures similar to Fig. 6 (not shown) we have verified this to be the case. The overall effect is a reduction of the effect of diffusion closer to the He flash.

\section{7. $\mu$ gradient inversion, diffusion and ${ }^{3} \mathrm{He}$}

The effects of concentration variations on $\mu$ as the $\mathrm{H}$ burning shell crosses the region of the concentration discontinuity left by the first dredge-up, are looked at in detail in Figs. 7 and 8. Consider $\Delta\left(1 / \mu_{0}\right)$ (where $\mu_{0}$ is the reduced mass per nucleus, that is excluding electrons) defined as the difference between the value of $1 / \mu_{0}$ at a given time step from that at the time step immediately after the first dredge-up; it measures the extent of the $\mu$ inversion. In the upper panel of Fig. 7 , at the maximum of $1 / \mu_{0}$ the value of $\Delta\left(1 / \mu_{0}\right)$ in the model without diffusion (dotted red curve) is a factor of 4.1 smaller than in the model with diffusion (solid red curve). Since the model with diffusion and the model without diffusion are affected in the same way by ${ }^{3} \mathrm{He}$ burning,

\footnotetext{
8 The effect of a steep He gradient of the drift velocity of metals is described in slightly more detail in the second paragraph of Sect. 3.1.3. of Richard et al. (2001).
}

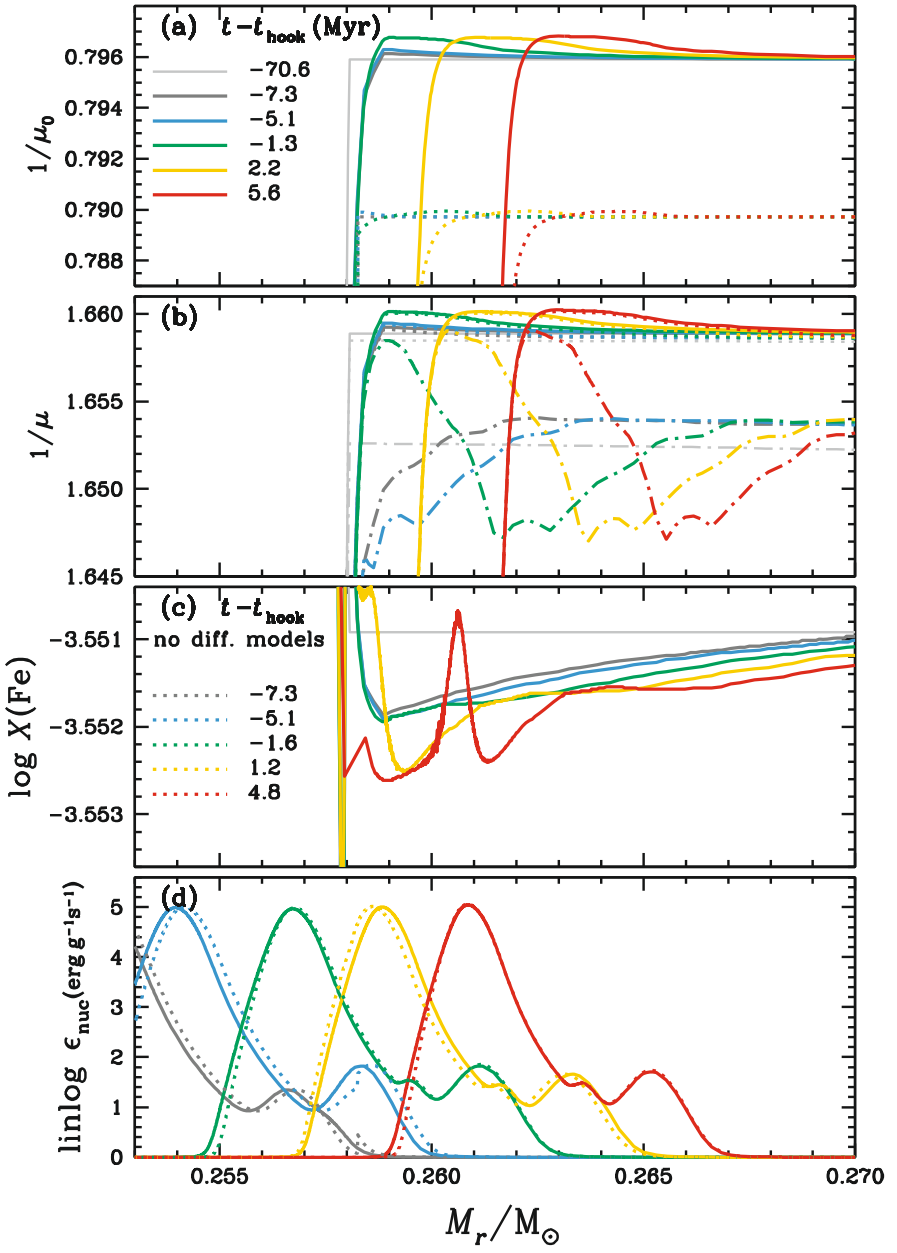

Fig. 7. Panel a) Inverted $\mu_{0}$ profiles in the model with diffusion (solid lines) and the model without diffusion (dotted lines) at a few time steps. Panel b) Inverted $\mu$ profiles in the model with diffusion assuming complete ionization (solid lines), ionization from OPAL tables (dot-dashed lines) and ionization from OPAL tables except $\mathrm{H}$ completely ionized (dotted lines). Panel c) Fe concentration in the model with diffusion. Note that the structures of the profiles move with the profiles of energy generation shown in panel d. Panel d) Nuclear energy generation profiles in the model with diffusion and in that without diffusion. Color coding identifies time steps in all panels of this figure and of Fig. 8.

this shows that the main cause of $\mu_{0}$ inversion when diffusion is properly taken into account is atomic diffusion.

In panel $b$, are shown the values of $1 / \mu$ in a model with diffusion calculated assuming complete ionization (solid lines). The dot-dashed lines were calculated using ionization from OPAL tables. The dotted lines were also calculated using ionization from OPAL tables except that $\mathrm{H}$ was assumed completely ionized showing that it is mainly responsible for the difference between $1 / \mu$ calculated assuming complete ionization and ionization from OPAL tables. The large variation of $1 / \mu$ around the ${ }^{3} \mathrm{He}$ burning region shows that one must carefully calculate ionization if one is to use small $\mu$ inversions to calculate instabilities.

The advancing burning shell on the bottom panel of Fig. 7 can be linked to the advancing $\mu_{0}$ inversion of panel a and the advancing structures of $\mathrm{Fe}$ concentration (panel c). These are not spurious but are explained by the analysis of Figs. 5 and 6 in Sect. 6. Fe underabundances precede the burning front (see the continuous dark grey curve). The effects of atomic diffusion on 
the concentration of Fe and other atomic species and so on $\mu_{0}$ can be perceived with difficulty on the continuous dark gray line of panel a because of the limited resolution of the latter. They are studied in more detail in Fig. 8. For that study, it is important that, as one may note from the bottom panel, most of the models represented by lines of similar color, one with diffusion (solid) and one without (dotted), are at very nearly the same evolutionary phase.

One may use Fig. 8 to analyze the roles of the atomic diffusion of metals and $\mathrm{He}$ as well as of ${ }^{3} \mathrm{He}$ burning in causing small $\mu$ inversions. The original discontinuity in the $1 / \mu$ curve was left over by the first dredge-up; it comes from previous nuclear evolution and diffusion, mainly during main-sequence evolution. One notes on the left panel $b$, the break in $X(\mathrm{Fe})$ created by the mixing of the Fe concentration profile originally caused by diffusion; it is absent on the right panel $b$, where diffusion is not taken into account. For $\mathrm{H}$ and $\mathrm{He}$ the discontinuities in the concentration profiles come from both diffusion and nuclear reactions and are so present in both the model with diffusion and that without diffusion.

Proportionately, Fe and He concentrations are affected by diffusion by similar factors but since He is some $50 \times$ more abundant than metals, its variation is the main cause of variations of $\mu$. Hydrogen and He are even more affected than Fe by diffusion, since the settling of the latter is reduced by $g_{\mathrm{rad}}(\mathrm{Fe})$ (see Figs. 5 and 6$)^{9}$. Left of the vertical line, the variation of $\mu$, at the age represented by the green line, $(-1.3 \mathrm{Myr})$ is mainly caused by $\mathrm{H}$ burning while right of it, it is caused by both atomic diffusion and ${ }^{3} \mathrm{He}$ burning. One notes in panel a that $\mu$ decreases by a larger factor (or $\Delta(1 / \mu)$ has a larger maximum) in the model with diffusion and that the changes occur there earlier than in the model without diffusion. Indeed a careful comparison of the right and left c and/or e panels shows that the change of $X(\mathrm{H})$ and $X(\mathrm{He})$ are first caused by atomic diffusion since, in the interval $0.259<M_{\mathrm{r}} / M_{\odot}<0.270$, the dotted dark gray lines for $\mathrm{H}$ and $\mathrm{He}$ (right panels) are horizontal while the corresponding full lines have respectively a maximum (left panel e) and a minimum (left panel c) where they cross the vertical line; the increase of the $\mathrm{H}$ abundance between the bottom of the convection zone and the vertical line is caused by atomic diffusion. At that age, ${ }^{3} \mathrm{He}$ burning does contribute additional $\mathrm{H}$ but only for smaller values of $M_{\mathrm{r}} / M_{\odot}$. It is only with the blue line that ${ }^{3} \mathrm{He}$ burning makes any dent on the $\mathrm{H}$ and ${ }^{4} \mathrm{He}$ concentrations beyond $M_{\mathrm{r}} / M_{\odot}=0.258$. With the dotted green line the effect of ${ }^{3} \mathrm{He}$ reaches $M_{\mathrm{r}} / M_{\odot}=0.262$. Its effect is clearly seen on the right panels $\mathrm{c}$ and e. It leads to a maximum of $X(\mathrm{H})$ at $M_{\mathrm{r}} / M_{\odot} \sim 0.26$ (see the green dotted curve). This implies that the diffusion of metals and $\mathrm{He}$ are the first to cause a reduction of $\mu$ and, later, the nuclear reactions involving ${ }^{3} \mathrm{He}$ add a contribution. In the model with diffusion, the effect is smaller for ${ }^{4} \mathrm{He}$ but larger for $\mathrm{H}$ since for $\mathrm{He}$ the effects of diffusion and ${ }^{3} \mathrm{He}$ burning partly cancel while they add for $\mathrm{H}$.

\subsection{Concentration gradients vs turbulence}

The preceding results were obtained without any adjustable parameters. The instability created by the $\mu$ inversion can be simulated by turbulent diffusion. It remains to determine what value of the turbulent diffusion coefficient startsmodifying the results

\footnotetext{
9 This is an example where the inclusion of the gravitational settling of metals improves the accuracy of the calculations only if $g_{\text {rad }}$ are also included.
}

obtained above. Do the $D_{\mathrm{T}}$ suggested for instance by Charbonnel \& Zahn (2007a) modify substantially the small concentration gradients of $\mathrm{He}$ and of metals caused by diffusion?

In first approximation, one may write the diffusion velocity equation in the form:

$v_{D}=\left(D_{i p}+D_{\mathrm{T}}\right)\left[-\frac{\partial \ln X_{i}}{\partial r}\right]+D_{i p}\left[\frac{A_{i} m_{p}}{k T}\left(g_{\mathrm{rad}, i}-g\right)+\ldots\right]$.

Within the first brackets on the right is the purely diffusive term which includes a contribution both from atomic diffusion, $D_{i p}$, and from turbulent diffusion, $D_{\mathrm{T}}$, which is generally assumed to be the same for all elements. In stellar evolution calculations we use a much more complete treatment of atomic diffusion than allowed by Eq. (2) and turbulent transport is then introduced by simply adding a pure diffusive term

$-D_{\mathrm{T}} \frac{\partial \ln X_{i}}{\partial r}$

to each element's diffusion velocity, which tends to reduce its abundance gradient in a way very similar to the effect of $D_{\mathrm{T}}$ in Eq. (2).

The concentration gradients of metals and of $\mathrm{He}($ or $\mathrm{H})$ are very small, yet it is only through those gradients that turbulence $\left(D_{\mathrm{T}}\right)$ has an effect, whereas atomic diffusion also acts through the much larger $g$ and $g_{\text {rad }}$ driving terms. Consequently, $D_{\mathrm{T}}$ can have an effect only if it is much larger than the atomic diffusion coefficient, $D_{i p}$. But how much larger? To have a significant effect on the diffusion velocity, $D_{\mathrm{T}}$ must lead to a contribution similar to the driving terms of atomic diffusion in Eq. (2). Turbulence then has an effect if

$D_{\mathrm{T}}\left|\frac{\partial \ln X_{i}}{\partial r}\right| \sim D_{i p}\left|\frac{A_{i} m_{p}}{k T}\left(g_{\mathrm{rad}, i}-g\right)\right|$.

Equation (4) was evaluated roughly using drift velocities of He (similar to those for metals shown on the middle panels of Fig. 5) and the value of $X(\mathrm{He})$ shown on the left panel c of Fig. 8. The $D_{\mathrm{T}}$ so obtained from Eq. (4) is shown in Fig. 9 where the color coding is the same as in Fig. 8. It varies between $10^{6}$, immediately above the burning shell, and $10^{7} \mathrm{~cm}^{2} / \mathrm{s}$ immediately below the surface convection zone.

The mixing caused by $\mu$ gradient inversion is a complex process (see for instance Proffitt 1989) leading to a non-linear diffusion equation since $D_{\mathrm{T}}$ is proportional to the inverted $\mu$ gradient. Following Proffitt's formalism, Proffitt \& Michaud (1989) give an approximation to the mixing (their Eqs. (2) to (5)) which is approximately equivalent to the values obtained by Kippenhahn (1974). However, existing evaluations of $D_{\mathrm{T}}$ in the literature, vary by orders of magnitude (see Vauclair 2004; Denissenkov \& Pinsonneault 2008; Théado et al. 2009 for recent discussions) mainly because there is no agreement on the size and shape of blobs or fingers. In their Fig. 2, Charbonnel \& Zahn (2007a), using elongated fingers, obtain $D_{\mathrm{T}}$ varying from $10^{5}$ close to the burning shell to $10^{10} \mathrm{~cm}^{2} / \mathrm{s}$ just below the surface convection zone; as mentioned in the last paragraph of their Sect. 2, this is two orders of magnitude larger than the estimate of Kippenhahn et al. (1980). They also give corresponding $\mathrm{d} \ln \mu / \mathrm{d} \ln P$ as of order $10^{-5}$ (see their Fig. 1). We have verified that the $\mathrm{d} \ln \mu / \mathrm{d} \ln P$ corresponding to the concentration gradients used to obtain our Fig. 9 are of order $10^{-4}$ or some ten times larger. From Eq. (4) reducing the $\mu$ gradient by a factor of 10 increases $D_{\mathrm{T}}$ by a similar factor or between between $10^{7}$ and $10^{8} \mathrm{~cm}^{2} / \mathrm{s}$. Given that these numbers are within the range of those obtained by Charbonnel \& Zahn (2007a), the settling of ${ }^{4} \mathrm{He}$ could be just as important as ${ }^{3} \mathrm{He}$ burning in creating instabilities between the $\mathrm{H}$ burning shell and the surface convection zone. 

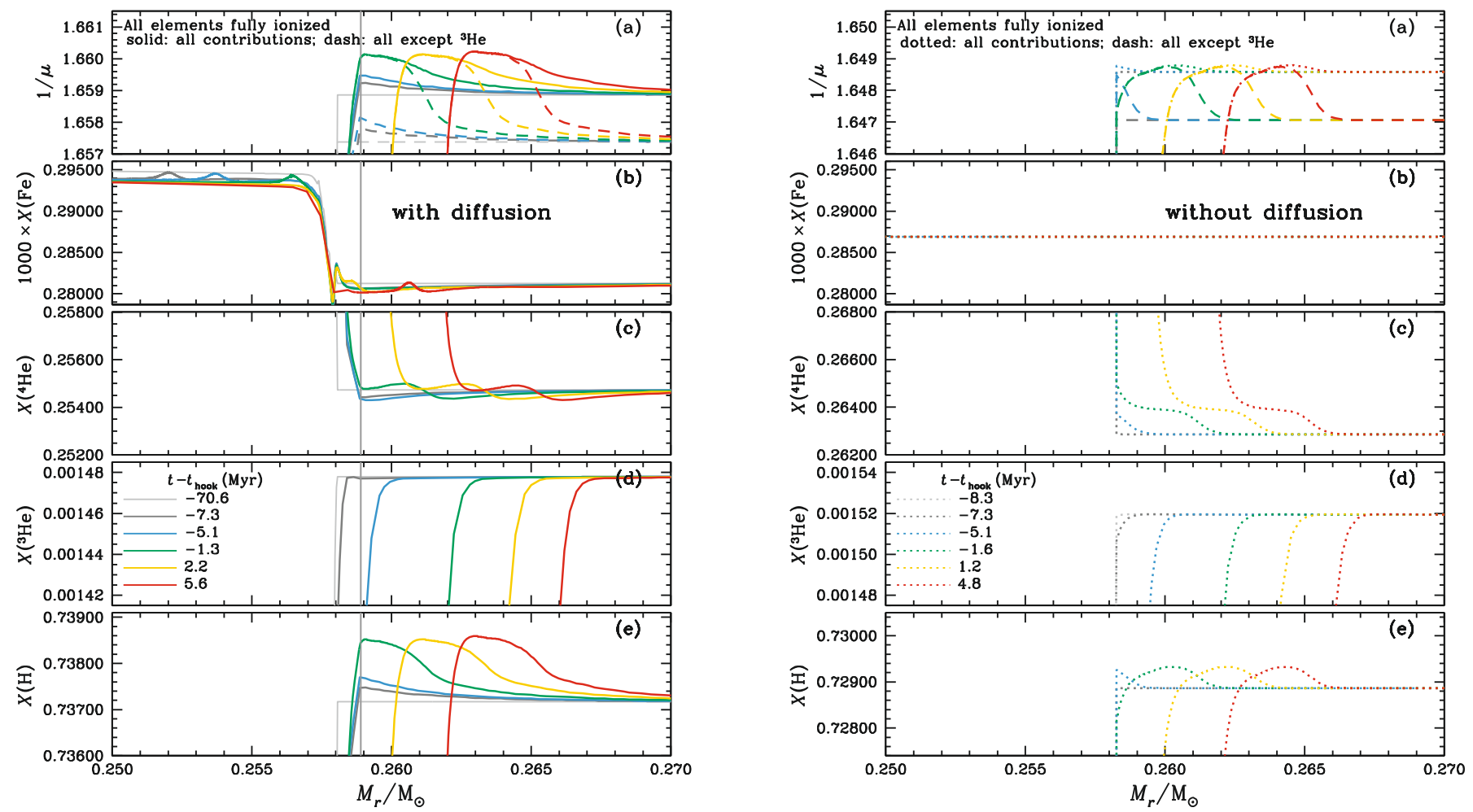

Fig. 8. Upper left panel Interior profile of $\mu$ for the model with diffusion immediately after the first dredge-up (light gray lines on the left panel) and at a few additional time steps (labeled by the same color code as used in Fig. 7) as the $\mathrm{H}$ burning shell crosses the region of the composition break left by the first dredge-up. Upper right panel same as left panel except for the model without diffusion; the curve immediately after the dredge-up is not shown since it is indistinguishable from the dotted dark-gray line. Complete ionization was imposed in calculating $\mu$ in order to avoid variations of $\mu$ caused by partial ionization (see Fig. 7). In the upper left panel, $\mu$ is shown by full lines. To obtain the short dashed curves, the ${ }^{3} \mathrm{He}$ contribution was removed from $1 / \mu$. The $\mathrm{Fe}, \mathrm{H},{ }^{3} \mathrm{He}$ and ${ }^{4} \mathrm{He}$ profiles are shown on a linear scale to emphasize how similar the $\mathrm{H}$ and $1 / \mu$ profiles are. For the two gray and the blue curves, left of the vertical line on the left panels, the variation of $\mu$ is caused by $\mathrm{H}$ burning while, right of it, it is caused by both atomic diffusion and ${ }^{3} \mathrm{He}$ burning.

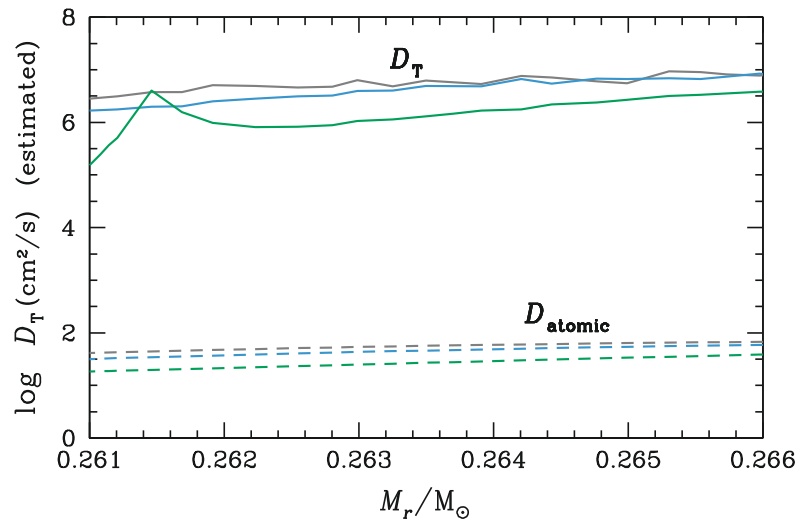

Fig. 9. Turbulent diffusion coefficient required to reduce significantly the effect of atomic diffusion as determined from Eq. (4). The color coding is the same as used in the left panels of Fig. 8. Above the burning shell, turbulent diffusion coefficients need to be at least $\sim 10^{6}$ to $10^{7} \mathrm{~cm}^{2} / \mathrm{s}$ to reduce by a factor of order 2 the gradients created by atomic diffusion. The atomic diffusion coefficient shown is that between $\mathrm{H}$ and $\mathrm{He}$.

\section{Conclusion}

One would not a priori expect large effects of atomic diffusion in RGB stars. Evolution through that phase is relatively rapid and gravity is small in the atmosphere implying that distances are relatively large so that diffusion is unlikely to have time to modify element concentrations. Surface abundances are not modified by diffusion on the RGB. But while it is true that gravity is small at the surface of RGB stars, it is large around the H burning shell. That is where atomic diffusion ends up playing a role. It was studied most carefully around the luminosity bump (see Sect. 6). In Fig. 6 the drift velocity of metals is shown to be nearly equal to the velocity of the $\mathrm{H}$ burning front. Consequently atomic diffusion can modify their concentration. Perhaps the greatest surprise of the results is the relatively important role played by $g_{\mathrm{rad}}$, so that the concentration of each metal is influenced differently by atomic diffusion which leads to slightly different concentration profiles for each metal within the core (see the upper panels of Fig. 5).

The effect on the observable properties of RGB stars are however very small. The most visible effect is at the so called bump region of the RGB (see the inset in Fig. 1). The He core mass at the flash of the model with diffusion is slightly larger than that of the model without diffusion but the difference is admittedly small: $\sim 0.003 M_{\odot}$ for the lowest metallicity considered, $Z=0.0001$, to $\sim 0.002 M_{\odot}$ at solar metallicity.

The $\mu$ inversion caused by ${ }^{3} \mathrm{He}$ burning past the luminosity bump was suggested to be the main cause of mixing between the $\mathrm{H}$ burning shell and the surface convection zone (Eggleton et al. 2006, 2008; Charbonnel \& Zahn 2007a). The gravitational settling of He between the surface convection zone and the $\mathrm{H}$ burning shell has been found to lead to a $\mu$ gradient inversion larger by a factor of $\sim 4.1$ than the $\mu$ inversion ${ }^{3} \mathrm{He}$ burning leads to (see Sect. 7). It has also been shown to occur slightly earlier during evolution. However the inversion created by ${ }^{3} \mathrm{He}$ burning is replenished on ${ }^{3} \mathrm{He}$ burning time scale whereas the settling of $\mathrm{H}$ is determined by the atomic diffusion time scale. 
The results presented in this paper were obtained entirely from first principles. We do not wish to imply that there is no role for turbulence either from differential rotation or related to the appearance of inverse $\mu$ gradients. However the comparison of the turbulent diffusion coefficients required to wipe out the effects of diffusion on the RGB (see Sect. 7.1) puts limits on the values of turbulent transport and on its causes. Atomic diffusion could be the main cause of the instability generated by an inverted $\mu$ gradient.

As mentioned at the end of Sect. 6, settling becomes less important as the star approaches the He flash. Consequently the mixing He settling can lead to is also progressively reduced past the luminosity bump. This appears consistent with the observational result that mixing on the RGB seems to occur mainly immediately past the bump (see the Introduction of Denissenkov \& VandenBerg 2003).

Acknowledgements. This research was partially supported at the Université de Montréal by NSERC. We thank the Réseau québécois de calcul de haute performance (RQCHP) for providing us with the computational resources required for this work. We thank Don VandenBerg for useful discussions and a careful reading of the manuscript and Santi Cassisi for his constructive review of the paper.

\section{References}

Cassisi, S., degl'Innocenti, S., \& Salaris, M. 1997, MNRAS, 290, 515

Cassisi, S., Castellani, V., degl'Innocenti, S., et al. 1998, A\&AS, 129, 267
Charbonnel, C., \& Zahn, J.-P. 2007a, A\&A, 467, L15

Charbonnel, C., \& Zahn, J.-P. 2007b, A\&A, 476, L29

Denissenkov, P. A., \& Pinsonneault, M. 2008, ApJ, 684, 626

Denissenkov, P. A., \& VandenBerg, D. A. 2003, ApJ, 593, 509

Eggleton, P. P. 1971, MNRAS, 151, 351

Eggleton, P. P., Dearborn, D. S. P., \& Lattanzio, J. C. 2006, Sci, 314, 1580

Eggleton, P. P., Dearborn, D. S. P., \& Lattanzio, J. C. 2008, ApJ, 677, 581

Kippenhahn, R. 1974, in Late Stages of Stellar Evolution, ed. R. J. Tayler, \& J. E. Hesser, 20

Kippenhahn, R., Ruschenplatt, G., \& Thomas, H.-C. 1980, A\&A, 91, 175

Michaud, G., Richer, J., \& Richard, O. 2007, ApJ, 670, 1178

Michaud, G., Richer, J., \& Richard, O. 2008, ApJ, 675, 1223

Proffitt, C. R. 1989, ApJ, 338, 990

Proffitt, C. R., \& Michaud, G. 1989, ApJ, 345, 998

Proffitt, C. R., \& VandenBerg, D. A. 1991, ApJS, 77, 473

Richard, O., Michaud, G., \& Richer, J. 2001, ApJ, 558, 377

Richard, O., Michaud, G., Richer, J., et al. 2002, ApJ, 568, 979

Richer, J., Michaud, G., Rogers, F., et al. 1998, ApJ, 492, 833

Rogers, F. J., \& Iglesias, C. A. 1992a, ApJS, 79, 507

Rogers, F. J., \& Iglesias, C. A. 1992b, ApJ, 401, 361

Rogers, F. J., Swenson, F. J., \& Iglesias, C. A. 1996, ApJ, 456, 902

Sweigart, A. V., \& Gross, P. G. 1978, ApJS, 36, 405

Sweigart, A. V., \& Mengel, J. G. 1979, ApJ, 229, 624

Théado, S., Vauclair, S., Alecian, G., et al. 2009, ApJ, 704, 1262

Turcotte, S., Richer, J., Michaud, G., Iglesias, C., \& Rogers, F. 1998, ApJ, 504, 539

Ulrich, R. K. 1972, ApJ, 172, 165

VandenBerg, D. A. 1992, ApJ, 391, 685

VandenBerg, D. A., Swenson, F. J., Rogers, F. J., Iglesias, C. A., \& Alexander, D. R. 2000, ApJ, 532, 430

Vauclair, S. 2004, ApJ, 605, 874

Zahn, J.-P. 1992, A\&A, 265, 115 